\title{
Genre, normes et langages du costume
}

Sophie Cassagnes-Brouquet et Christine Dousset-Seiden

\section{Q OpenEdition \\ Journals}

Édition électronique

URL : http://journals.openedition.org/clio/10714

DOI : 10.4000/clio. 10714

ISSN : 1777-5299

Éditeur

Belin

Édition imprimée

Date de publication : 31 décembre 2012

Pagination : 7-18

ISSN : 1252-7017

\section{Référence électronique}

Sophie Cassagnes-Brouquet et Christine Dousset-Seiden, «Genre, normes et langages du costume», Clio. Femmes, Genre, Histoire [En ligne], 36 | 2012, mis en ligne le 07 mars 2013, consulté le 10 décembre 2020. URL : http://journals.openedition.org/clio/10714; DOI : https://doi.org/10.4000/clio. 10714 


\title{
Genre, normes et langages du costume
}

\author{
Sophie CASSAGNES-BROUQUET \& \\ Christine DOUSSET-SEIDEN
}

Longtemps dédaigné par les historiens, le costume fait aujourd'hui l'objet d'un retour en force dans les études historiques et sociologiques ${ }^{1}$. Élément essentiel de la culture matérielle, il est l'un des marqueurs de toute société humaine, véritable langage destiné à signifier et pas seulement à protéger ou à orner ${ }^{2}$. Le costume, mais aussi la façon de le porter, de le regarder et de l'interpréter, sont les reflets des normes sociales. Comme le souligne Daniel Roche: "l'histoire du vêtir témoigne en profondeur sur les civilisations. Elle en révèle les codes $»^{3}$.

C'est une évidence que de rappeler combien le costume est indissociable des conditions socioculturelles dans lesquelles il se porte. Affirmation de soi, il doit pourtant nécessairement rencontrer le regard de l'autre. Par sa visibilité, il est alors conçu comme un message, collectif ou individuel, conscient ou inconscient, que celui qui le revêt s'adresse à lui-même et aux autres ${ }^{4}$. La littérature et l'art ne s'y trompent pas qui, depuis l'Antiquité jusqu'à nos jours, lui accordent une fonction essentielle de marquage social, mais jouent aussi sur ses mensonges et ses travestissements 5 .

Il ne se résume donc pas aux phénomènes de mode et endosse bien d'autres dimensions: symboliques, culturelles, religieuses, sociales et politiques. Adhésion ou critique sociale, il s'affiche comme

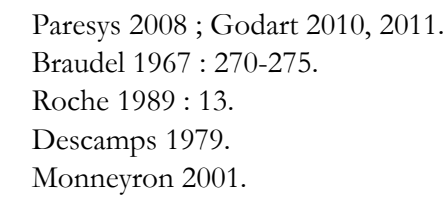


le moyen le plus simple et souvent le plus efficace pour s'inscrire dans le paysage humain. Le costume est politique comme le montre, parmi tant d'autres exemples, la période révolutionnaire avec ses sansculottes ou le vêtement féminin sous le Directoire, à la grecque ou à la romaine, qui, tout à la fois, libère le corps féminin et propose des modèles républicains. Il n'est donc jamais neutre ni secondaire, il sert les hiérarchies ou les combat. Il signe un statut, une fonction, par exemple quand il se fait uniforme. Il peut aussi constituer un facteur d'intégration dans un groupe ou une classe d'âge comme celle de l'adolescence. Il révèle l'individualité ou cherche à la nier comme le costume religieux ou militaire. Entre suivisme ou conformisme, uniformisation et pourquoi pas mondialisation, il peut aussi se faire revendication de son unicité.

Devenu «territoire de l'historien", mais aussi de bien d'autres sciences humaines, histoire de l'art, anthropologie, sociologie, psychologie, philosophie et linguistique, le costume trouve donc tout naturellement sa place dans une réflexion sur le genre. C'est en ce sens que nous avons préféré le terme de costume à celui de vêtement, car celui qui l'endosse s'apprête, tel un comédien, à jouer un rôle choisi ou imposé sur la scène historique et sociale. Vêtement est un terme plus neutre comme le signale l'Encyclopédie dans l'article qu'elle lui consacre, où l'auteur souligne sa fonction première de protection du corps ou d'ornement - «tout ce qui sert à couvrir le corps, à l'orner, ou le défendre des injures de l'air ». Aussi il lui préfère le mot « costume », venu d'Italie au XVIIe siècle, dont l'étymologie évoque la coutume tout comme l'habit, l'habitus ${ }^{6}$.

Construction sociale et culturelle, le costume dévoile autant qu'il masque la réalité des groupes et des individus. Il intègre ou exclut et prend une part essentielle dans la fabrique du genre ${ }^{7}$.

\section{Histoire et sociologie du costume}

Le costume a ses historiens depuis plus de deux siècles. Au début du XVIII ${ }^{e}$ siècle, Bernard de Montfaucon le définissait déjà comme un élément participant à l'écriture de l'histoire. Dans ses Monumens de la

Roche 1989 : 12 ; Boutin-Arnaud \& Tasmadjian 1997.

7 Pellegrin 1993. 
monarchie françoise, avec les figures de chaque règne que l'injure du temps a épargnées, la représentation des vêtements est abondamment illustrée en tant que composante de l'histoire française, reconnue comme source au même titre qu'un document écrit ou un objet archéologique. À partir du milieu du XIX ${ }^{\mathrm{e}}$ siècle, des auteurs se passionnent pour le costume, et entreprennent la rédaction d'ouvrages encyclopédiques comme Jules-Étienne Quicherat (18141882) ${ }^{8}$. Albert Racinet (1825-1893) a l'idée d'écrire une histoire mondiale du costume qui demeure l'ouvrage le plus détaillé et le plus complet dans ce domaine 9 . Maurice Leloir (1853-1940) publie entre 1933 et 1938 L'Histoire du costume de l'Antiquité à 191410 et fonde en 1907 la Société de l'Histoire du Costume dans le but d'encourager les recherches et de créer un musée à Paris. Il lègue sa collection en 1920 à la Ville de Paris qui l'intègre au musée Carnavalet. Le musée du Costumes de la Ville de Paris est inauguré en 1956 rue du Président Wilson, puis transféré au Palais Galliera en 1977. La Société change alors de nom pour devenir la Société de l'Histoire du Costume-Amis du musée Galliera. Peu à peu, un savoir se construit en un processus qui permet la constitution d'un corpus de sources écrites, iconographiques et matérielles. Une historiographie se développe parallèlement à la multiplication des expositions dans les années 1970, une dynamique qui s'intensifie à partir des années 1990. Cette collecte s'accompagne d'une réflexion sur le costume et l'idée se dégage de son caractère éminemment culturel et historique.

L'histoire du costume en France et en Europe occidentale est relativement bien traitée. Considérée de façon partielle à la fin du XIX siècle à travers des ouvrages historiques généraux - le Dictionnaire de Viollet-le-Duc, le Manuel d'archéologie de Camille Enlard, le Glossaire archéologique de Victor Gay -, elle fait l'objet d'une étude complète au début du XXe siècle, initiée d'un côté par l'ethnologie afin d'analyser la production des textiles contemporains et les costumes folkloriques, et de l'autre par l'archéologie pour l'étude des civilisations anciennes. Les sciences humaines s'emparent du costume dans le but d'étendre les

\footnotetext{
Quicherat 1875-1877.

Racinet 1875-1888.

10 Leloir 1933.
} 
connaissances sur la vie artistique et culturelle, la sociologie, l'économie et l'histoire des techniques.

Si les auteurs de la fin du XIXe et du début du XXe siècle se sont principalement appliqués à composer des encyclopédies, il faut attendre les années 1960 pour que les philosophes et les sociologues s'intéressent au costume. Roland Barthes est le premier dans son article fondateur «Histoire et sociologie du vêtement. Quelques observations méthodologiques", paru dans les Annales en 195711. Dans les années 1990, historiens et historiens de l'art multiplient les études sur la mode ancienne et actuelle comme Daniel Roche avec son ouvrage fondateur La culture des apparences. Une histoire du vêtement, $X V I I^{e}-X V I I I^{e}$ siècle. Biographies, monographies et catalogues viennent compléter la bibliothèque du patrimoine textile. Enfin, de véritables réseaux d'étude à vocation universelle se créent dont le premier est COSTUME, cellule de l'ICOM (International Council of Museums) dédiée au patrimoine textile, créée lors de la $6^{\mathrm{e}}$ Conférence générale de l'UNESCO en 1962. La revue électronique Apparences, créée en 2007 sous l'égide d'Isabelle Paresys, témoigne de cette volonté de recherche pluridisciplinaire ${ }^{12}$, tout comme les nombreux colloques et expositions qui se sont déroulés au cours des dernières années tels l'exposition Fastes de cour et cérémonies royales, le costume de cour en Europe, 1650-1800 et le colloque tenu conjointement ${ }^{13}$, ainsi que le séminaire d'Histoire de la mode et du vêtement organisé cette année par l'Institut national d'Histoire de l'Art. Source d'histoire et d'histoire de l'art, le vêtement est aussi appréhendé par les sociologues et anthropologues comme un marqueur identitaire.

\section{Pour une histoire genrée du costume}

La définition des normes de genre à travers le costume et les discours qu'il suscite, leurs transformations, les contestations dont elles font l'objet, constituent évidement un élément important de la réflexion actuelle. Le costume participe à la construction d'une identité de

11 Barthes 1957.

12 La revue est publiée par l'IRHiS - Institut de Recherches Historiques du Septentrion (CNRS, UMR 8529 - Université Lille 3) http://irhis.recherche.univ-lille3.fr

13 Paresys \& Coquery 2001. 
genre, tantôt revendiquée, tantôt imposée ou refusée, et toujours variable selon l'âge, le statut ou le rang. Dans ce processus, doivent être prises en compte sa fonction érotique ou, au contraire, la volonté de désexualiser ou de masquer le corps qu'il concrétise. L'association ou la confrontation par le costume entre identité de genre et identités sociales, politiques ou religieuses ouvrent un vaste champ d'enquête, de la policière en uniforme à la femme en niqab pour se limiter à des exemples contemporains. Le costume européen, mais aussi celui des autres civilisations, peut également exprimer la tension entre l'occidentalisation et son refus ou bien l'habile mariage des deux. Sans cesse, le costume s'adapte, se modifie et évolue pour répondre aux sollicitations sociales. Ces transformations représentent en amont d'importants enjeux économiques, que l'on pense au poids de l'industrie textile dans les économies anciennes ou à celui de l'industrie de la mode aujourd'hui.

Par le jeu des formes et des accessoires, par la gestuelle qu'il autorise, le vêtement cache le corps ou au contraire le modèle en mettant en valeur certaines de ses parties pour mieux exprimer féminité ou masculinité selon des canons de beauté genrés, qui évoluent au fil du temps. Formes, couleurs, textures sont autant de signifiants qui semblent évidents en matière de genre, cependant, à bien y regarder, les choses ne sont pas si simples comme en témoignent les doutes récemment émis par les archéologues sur une attribution trop facilement sexuée des éléments de la parure. La contribution de Chloé Belard sur les ceintures à l'âge du Fer en Champagne apporte un éclairage sur ces questions épistémologiques. Au premier abord, la distinction semble évidente entre les ceintures d'hommes, destinées à suspendre l'épée ou le poignard et celles des femmes, simples accessoires vestimentaires et ornementaux. Pourtant, la détermination du sexe du défunt par l'anthropologie biologique permet de nuancer cette dualité. Par ailleurs l'emplacement de la ceinture dans la tombe n'indique pas qu'elle était la propriété du défunt. Les ornements ne peuvent donc être considérés comme un «marqueur sexuel » automatique.

Jusqu'à l'époque contemporaine, ce sont le plus souvent les discours ou les représentations qui assignent au vêtement une identité de genre comme le montre Florence Gherchanoc à propos de la 
Grèce antique qui oppose ainsi le corps féminin couvert au corps masculin nu ${ }^{14}$. De manière assez invariante, depuis l'Antiquité jusqu'à l'époque contemporaine, les discours élaborés par les poètes, les historiens, les moralistes, les législateurs se concentrent volontiers sur le vêtement féminin pour le critiquer ou le contrôler. Ils mettent en avant l'intérêt des femmes à orner leurs corps pour répondre aux canons de la féminité tout en soulignant le statut social de leurs maris ou pères, mais ils témoignent également d'une méfiance envers le corps paré de la femme, source de ruse et d'érotisme ${ }^{15}$. Le costume doit être conforme à un comportement défini par les hommes selon des critères qui varient assez peu en définitive au fil de l'histoire : la condition, l'âge et les lieux ${ }^{16}$.

Odile Blanc dans Parades et parures. L'invention du corps de mode à la fin du Moyen Âge a bien montré comment le vêtement masculin est le premier au Moyen Âge à expérimenter un nouveau rapport au corps, et pourtant le discours préfère se focaliser sur le costume féminin, beaucoup moins audacieux ${ }^{17}$. La « différence sexuelle inscrite au cœur des pratiques vestimentaires " constitue un rappel permanent de la faute d'Ève. La femme est coupable et sa parure est un redoutable instrument de séduction pour les hommes, victimes des femmes. La ruse féminine passe par la parure, le jeu du caché/montré, du voile ou du dévoilement; le vêtement féminin inquiète, il faut le brider. La contribution de Maria-Giuseppina Muzzarelli, consacrée aux coiffures féminines dans l'Italie de la Renaissance au travers du prisme des lois somptuaires, démontre ici cette obsession des autorités de contrôler ces attributs vestimentaires. Le costume masculin, beaucoup moins réglementé, est libre de dévoiler une identité sexuée : ajusté au corps, il dévoile une virilité triomphante comme l'a montré récemment une histoire de la braguette ${ }^{18}$.

Les sociétés se sont attachées à produire des costumes masculins, soulignant la virilité de ceux qui les revêtent et les dotant d'une identité

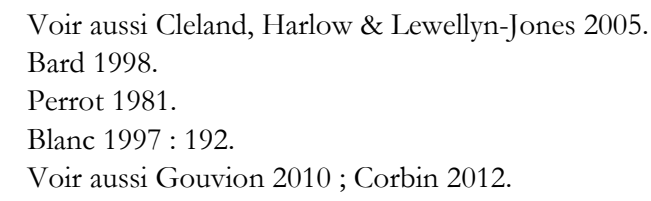


visuelle immédiate, porteuse d'autorité par le biais des uniformes comme le montre Odile Roynette dans sa contribution consacrée au costume militaire au XIX ${ }^{\mathrm{e}}$ siècle. L'armée et les forces de l'ordre constituent des laboratoires de la masculinité. L'uniforme fabrique une virilité idéale. Il nie l'identité individuelle au profit d'une communauté de genre comme le souligne également Arnaud Houte à propos de l'uniforme des forces de l'ordre dans la France du XIX ${ }^{\mathrm{e}}$ siècle ${ }^{19}$.

Ce sont encore ces discours et ces images produits par les sociétés contemporaines sur le vêtement que Roland Barthes a brillamment analysés dans son Système de mode en 1967 à travers l'exemple de la presse féminine ${ }^{20}$. L'analyse structurale met en avant la différence entre le vêtement réel, l'image et l'écriture ${ }^{21}$ par le biais d'un travail de codification sociale mais aussi d'a priori moraux ou psychologisants de types féminins comme la «délurée », la «piquante», la « sage», la " coquette », la «sportive », qui permettent l'identification ou le rêve aux lectrices. Une culture de mode s'impose et fait désormais l'objet d'un enseignement à l'Université de la mode de Lyon II, présentée par Martine Villelongue dans la rubrique Témoignages ${ }^{22}$.

Comme le souligne Roland Barthes, ce n'est donc pas seulement le vêtement, mais la façon dont il est porté et regardé qui importe. Catherine Baroin dans sa contribution consacrée aux codes vestimentaires romains souligne également cet aspect fondamental. Ce n'est que lorsqu'il est porté que le vêtement antique acquiert une connotation masculine ou féminine.

\section{Hiérarchiser, classer, exclure}

Instrument d'individualisation dans les sociétés occidentales contemporaines, le costume a davantage été au cours de l'Histoire, celui d'un contrôle et d'une hiérarchisation des sexes, des âges et des statuts sociaux. Le costume de cour, tel qu'il se développe à la fin du Moyen Âge pour connaître son apogée au XVIII e siècle, est un instrument politique au service du prince afin d'afficher son pouvoir

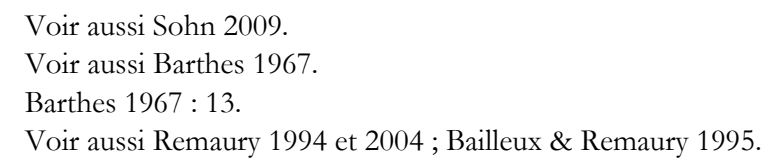


mais aussi de contrôler ses courtisans. Il joue un rôle hautement politique. La constitution de la garde-robe apparait comme un enjeu crucial pour le pouvoir dans le temps présent et pour la postérité, car elle accompagne l'image officielle du souverain. Chaque cour européenne adopte son costume, mais subit en même temps l'influence des puissances internationales comme celle de la France.

Cette volonté de contrôle du corps par des costumes aussi richement ornés que contraignants s'oppose, en apparence, à la simplicité des ordres ecclésiastiques faisant vœu de pauvreté. Cependant, la contribution de Christine Aribaud souligne combien la cérémonie de la vêture dans l'ordre des Carmélites met également en scène le rôle d'intégration du costume dans une communauté humaine, certes différente de la cour, mais qui n'en possède pas moins ses propres hiérarchies et la même volonté d'uniformisation et de contrôle des corps.

Cette tension entre le groupe et les individus se perçoit également à des niveaux sociaux plus modestes comme en témoigne la contribution de Marlène Albert-Llorca et Bénédicte Bonnemason à propos des costumes féminins traditionnels de la vallée d'Ossau. La mise en place d'un costume folklorique au XIX ${ }^{e}$ siècle dans cette vallée pyrénéenne s'est accompagnée d'une hiérarchisation assez nette entre le costume masculin, moins valorisé, et le costume féminin. Ce dernier est attaché au statut de la «maison » paysanne qui se concrétise dans la jupe rouge, celle que portaient autrefois les héritières.

Cette contrainte n'est pas seulement imposée aux femmes; souvent moins clairement exprimées, les pressions exercées sur le vêtement masculin n'en sont pas moins essentielles. Catherine Baroin montre combien la toge romaine signifie tout autant, sinon davantage, que la masculinité le statut de citoyen romain par opposition à l'esclave nu et se décline au fil des âges et des dignités. Le costume construit l'identité de genre, mais aussi l'identité sociale, tel le costume bourgeois, et de façon moins soulignée mais non moins réelle l'identité politique et l'identité religieuse, symbolique (pour signaler une posture morale, vêtements sombres des réformés, puritains, quakers, amish).

Plusieurs articles le soulignent: en tant que marqueur d'identité, le rôle du costume prend davantage d'importance à certains moments de la vie individuelle et collective. Endosser une nouvelle tenue est une façon 
plus ou moins ritualisée de marquer le passage d'un statut à un autre : de l'enfant au jeune, de la jeune fille à l'épouse, de la femme mariée à la veuve, du läque au religieux, du civil au soldat... Lors des fêtes, sa mise en scène permet de montrer et d'affirmer, voire de revendiquer, la place et le rôle de chacun et de chacune aux yeux de tous.

Si le costume classe, ordonne et hiérarchise la société, il permet aussi l'exclusion en devenant la marque d'infamie la plus visible et la plus aisément adoptée au cours des siècles. Depuis l'Antiquité jusqu'à une époque très récente, il oppose la femme honnête à celle de mauvaise vie. Dans l'Athènes classique, les hétaïres affichent un vêtement aisément reconnaissable par sa richesse et sa transparence ; objet de vives critiques, le costume devient l'indice d'un mauvais comportement et souvent d'une vie dépravée. Les premières lois somptuaires mettent en place les critères du costume honnête ou dépravé, mais il n'est pas imposé comme il peut l'être au Moyen Âge et dans l'Italie de la Renaissance, qui voient les règlements se multiplier afin d'imposer des couvre-chefs et des couleurs de tissus, en particulier le jaune, réservés aux prostituées et aux femmes appartenant aux communautés juives comme le souligne MariaGiuseppina Muzzarelli. L'exclusion passe souvent par l'apparence et, en particulier, par le costume à travers ses formes et ses couleurs.

\section{Transgression et travestissement}

Le costume peut également déjouer les codes et signifier la transgression. L'intérêt grandissant des historiens pour le travestissement a permis de mettre en avant ce jeu entre nature et culture, identité sexuée et identité de genre ${ }^{23}$. Sources écrites ou figurées témoignent d'une fascination pour l'inversion des costumes, largement utilisée dans la littérature. La transgression la plus évidente est d'adopter un vêtement appartenant au sexe opposé comme les chaperons adoptés par les femmes de l'Italie de la Renaissance au grand dam des réformateurs et des moralistes.

Elle peut être occasionnelle, imposée ou revendiquée comme en témoigne l'autoportrait de Frida Kahlo en 1940 Autorretrato con Pelo

23 Voir aussi Clio HFS, 1999, 10 ; Steinberg 2001. 
Cortado, présenté par Julie Crenn. L'artiste s'y représente assise sur une chaise, vêtue d'un costume masculin gris sombre, ses cheveux coupés jonchant le sol, mais portant une boucle d'oreille, sous les traits d'un être androgyne qui associe les genres. Cependant, plus que son costume, c'est sans doute sa façon de le porter qui est provocatrice comme le fit César dans la Rome antique. Vêtu de la toge du citoyen, Jules César n'en est pas moins dénoncé par plusieurs de ses contemporains pour la façon dont il ceinture sa toge et pour sa démarche molle qui constitue une transgression vestimentaire car elle s'oppose à la fermeté virile, ainsi que le montre Catherine Baroin.

Ces écarts vestimentaires permettent d'affirmer une singularité au sein d'un groupe social mais sont également porteurs d'un «trouble dans le genre " signalé, à propos de l'imagerie des femmes en uniforme, par Odile Roynette qui souligne le rôle de ce vêtement dans la valorisation d'une virilité guerrière.

L'apparence, vêtement et coiffure, de trois femmes britanniques dans l'Inde coloniale présentées ici par Arundhati Virmani, est aussi en rupture avec les stéréotypes de genre, qu'ils soient anglais ou indiens, par leur refus des attributs de la féminité et de la séduction. Mais leur choix est aussi politique ou philosophique comme le révèlent leurs parcours au sein des mouvements nationalistes et/ou spirituels indiens. Elles ont élaboré un costume à mi-chemin entre le sari traditionnel et le vêtement occidental, véritable métissage des cultures, mais un métissage inversé dans lequel les cultures dominées semblent s'imposer à celle des dominants. L'invention d'un vêtement proche d'un sari exprime sans ambiguïté les positions politiques de ces femmes et leur permet d'afficher leur différence et leurs affinités. Le vêtement devient ainsi un identifiant politique.

Objet évocateur de la frivolité, instrument central de la culture de mode, fragile et pourtant essentiel, le costume se révèle au fil des pages comme l'un des indicateurs les plus constants et les plus contraignants des différences de genre dont il est à la fois le révélateur et l'instrument. Hommes et femmes y projettent leurs aspirations mais aussi leurs conditionnements, parfois pour mieux se révolter et le jeter aux orties comme le firent les féministes américaines en 1968 
avec leur soutien-gorge dans une " poubelle de la liberté »24. Pantalons et jupes font aujourd'hui l'objet de débats de société et d'œuvres de fiction ${ }^{25}$, révélant des positions aussi intransigeantes que celles des législateurs et des moralistes de l'Antiquité ou de la Renaissance.

\section{Bibliographie}

Bailleux Nathalie \& Bruno Remaury (dir.), 1995, Modes et vêtements, Paris, Gallimard.

BARD Christine, 1998, Les garçonnes : modes et fantasmes des années folles, Paris, Flammarion.

—, 2010a, Une histoire politique du pantalon, Paris, Le Seuil.

—, 2010b, Ce que soulève la jupe : identités, transgressions, résistances, Paris, Éd. Autrement.

BARTHES Roland, 1957, «Histoire et sociologie du vêtement. Quelques observations méthodologiques », Annales, Économies, Sociétés, Civilisations, 3, p. 430-441.

—, 1967, Système de la mode, Paris, Le Seuil.

Blanc Odile, 1997, Parades et parures. L'invention du corps de mode à la fin du Moyen Âge, Paris, Gallimard.

Boutin-Arnaud Marie-Noëlle \& Sandrine TASMADJIAN, 1997, Le Vêtement, Paris, Nathan.

BRAUDEL Fernand, 1967, Civilisation matérielle et Capitalisme, t.1, Les structures du quotidien, Paris, Armand Colin.

Cleland Liza, Harlow Mary \& Lloyd Lewellyn-Jones (eds), 2005, The Clothed Body in Ancient World, Oxford, Oxbow Book.

CLIO, Histoire, femmes et sociétés, 10, 1999, "Femmes travesties : un "mauvais" genre ». Christine Bard \& Nicole Pellegrin (dir.)

CORBIN Alain, 2012, Histoire de la virilité, 2/ Le triomphe de la virilité, Paris, Le Seuil.

Descamps Marc Alain, 1979, Psychosociologie de la mode, Paris, PUF.

GODART Frédéric, 2010, Sociologie de la mode, Paris, La Découverte.

—, 2011, Penser la mode, Paris, Éd. du Regard.

Gouvion Colette, 2010, Braguettes, une histoire du vêtement et des mours, Rodez, Éditions du Rouergue.

24 Bard 2010.

25 Ibid. 
HéRITIER Françoise, 2010, Hommes, femmes : la construction de la différence, Paris, Le Pommier.

LeloIr Maurice, 1933-1949, Histoire du costume de l'Antiquité à 1914, Paris, Ernst Henri.

Lethuillier Jean-Pierre (dir.), 2009, Les costumes régionaux entre mémoire et histoire, Rennes, PUR.

Monneyron Frédéric, 2001, La Frivolité essentielle. Du vêtement et de la mode, Paris, PUF.

PARESYS Isabelle, 2008, Paraître et apparences en Europe occidentale du Moyan âge à nos jours, Villeneuve-d'Ascq, Presses universitaires du Septentrion.

PARESYS Isabelle \& Natacha COQUERY (dir.), 2011, Se vêtir à la cour en Europe, 1400-1815, Villeneuve-d'Ascq, Centre de recherche du Château de Versailles / Institut de Recherches Historiques du Septentrion de l'université Lille 3-Charles-de-Gaulle.

PeLLEGRIN Nicole, 1993, «Le vêtement comme fait social total», in Christophe CHARLE (dir.), Histoire sociale, histoire globale?, Paris, Fondation de la Maison des sciences de l'homme, p. 81-94.

Perrot Philippe, 1981, Les Dessus et les Dessous de la bourgeoisie. Une bistoire du vêtement au XIX siècle, Paris, Fayard.

QUiCHERAT Jules-Étienne, 1875-1877, Histoire du costume en France depuis les temps les plus reculés jusqu'à la fin du XVIII' siècle, Paris, Hachette.

RACINET Albert, 1875-1888, Le costume bistorique en cinq cents planches chromolithographiques, Paris, Firmin-Didot.

Remaury Bruno (dir.), 1994, Dictionnaire de la mode an XX'e siècle, Paris, Éd. du Regard.

—, 2004, Marques et récits : la marque face à l'imaginaire culturel contemporain, Paris, Éd. du Regard.

Roche Daniel, 1989, La culture des apparences. Une histoire du vêtement, XVII'-XVIII siècle, Paris, Fayard.

SOHN Anne-Marie, 2009, « Sois un homme ». La construction de la masculinité au XIXe siècle, Paris, Le Seuil.

STEINBERG Sylvie, 2001, La confusion des sexes. Le travestissement de la Renaissance à la Révolution, Paris, Fayard. 